\title{
Pneumomediastinum as a complication of fast bowling in cricket
}

\author{
M. R. CLEMENTS \\ B.SC., M.R.C.P.
}

\author{
D. V. HAMILTON \\ M.B., M.R.C.P.
}

Addenbrooke's Hospital, Cambridge CB2 $2 Q Q$

\begin{abstract}
Summary
The case is described of a 15-year-old patient who developed acute pneumomediastinum and bilateral pneumothoraces whilst playing cricket. He made a rapid and uncomplicated recovery.
\end{abstract}

\section{Introduction}

Pneumomediastinum is an uncommon cause of retrosternal chest pain in sportsmen. It has been described in two footballers who sustained chest-wall trauma (Morgan \& Henderson, 1981), and two mountain climbers (Vosk \& Houston, 1977), and we should like to report its recent occurrence in a young cricketer.

\section{Case report}

A 15-year-old schoolboy was practising bowling and straining to bowl fast, when he noticed the gradual onset of sharp retrosternal chest pain, aggravated by respiration and eased by lying down. Later the pain radiated to his shoulders and neck, which became swollen. On examination there was a loud pericardial rub and surgical emphysema around the neck and over the anterior chest-wall. There were no stigmata of Marfan's syndrome. Chest X-ray confirmed extensive surgical emphysema in the soft tissues of the neck and right axilla and showed a rim of air along the edge of the pericardium consistent with pneumomediastinum. Subsequent chest X-rays revealed small bilateral apical pneumothoraces which resolved uneventfully over the next three weeks.

\section{Discussion}

Pneumomediastinum is a rare condition which occurs spontaneously in neonates, sometimes follow- ing aspiration, and in fit young men. It has also been reported in association with childbirth, diabetic ketoacidosis, anorexia nervosa, Valsalva manoeuvres during marijuana smoking, asthma, lung function testing and sudden decompression. It is surprising that there have been so few reports of its occurrence during the acute exertion of athletic competition. The condition arises as a result of a sudden increase in the pressure within surface alveoli, which may occur spontaneously, probably as a result of check-valve bronchiolar obstruction, or during closed-chest trauma. Rupture of the alveoli allows free air to track along perivascular sheaths and enter the mediastinum at the hilum. Pneumothorax is a common accompaniment. Air may also enter the mediastinum following rupture of the oesophagus, or as an incidental extension of free air below the diaphragm from a perforated abdominal viscus. Pericardial pain is typical and dyspnoea is common and may be severe. A loud pericardial crunching or clicking sound best heard in the left lateral decubitus position is present in 50\% of cases (Hamman, 1937). However, it is not diagnostic and may be heard with a pneumothorax.

Treatment is invariably conservative. The condition is self-limiting and should be considered in the differential diagnosis of retrosternal chest pain, particularly if straining has occurred before the onset of pain.

\section{References}

Hamman, I. (1937) Spontaneous interstitial emphysema of the lungs. Transactions of the Association of American Physicians, 52 , 311.

Morgan, E.J. \& Henderson, D.A. (1981) Pneumomediastinum as a complication of athletic competition. Thorax, 36, 155.

Vosk, A. \& Houston, C.S. (1977) Mediastinal emphysema in mountain climbers. Heart and Lung, 6/5, 799. 